\title{
Effect of unlabeled helper probes on detection of an RNA target by bead-based sandwich hybridization
}

\author{
Kim Bundvig Barken¹, Magdalena Gabig-Ciminska²,3, Anders Holmgren², and Søren Molin ${ }^{4}$
}

BioTechniques 36:124-132 (January 2004)

\begin{abstract}
Unlabeled helper oligonucleotides assisting a bead-based sandwich hybridization assay were tested for the optimal placement of the capture and detection probes. The target used was a full-length in vitro synthesized mRNA molecule. Helper probes complementary to regions adjacent to the binding site of the $5^{\prime}$ end attached capture probe were found much more effective than helper probes targeting positions adjacent to the detection probe binding site. The difference is believed to be caused by a disruption of the RNA secondary structure in the area where the capture probe binds, thereby reducing structural interference from the bead. The use of additional helpers showed an additive effect. Using helpers at both sides of the capture and detection probes showed a 15-to 40-fold increase in hybridization efficiency depending on the target, thereby increasing the sensitivity of the hybridization assays. Using an electrical chip linked to the detection probe for the detection of p-aminophenol, which is produced by alkaline phosphatase, a detection limit of $2 \times$ $10^{-13} \mathrm{M}$ mRNA molecules was reached without the use of a nucleic acid amplification step.
\end{abstract}

\section{INTRODUCTION}

The effect of contiguously annealed modular primers has been extensively studied in DNA sequencing, where they have been used for priming polymerase. Kieleczawa et al. (1) initially suggested using contiguously annealed hexamers for DNA sequencing by primer walking for the creation of a pre-made library of primers. This method was later duplicated (2-5). The base stacking interaction between the $5^{\prime}$-terminal base of one module and the $3^{\prime}$-terminal base of the adjacently positioned module was one of the effects that made the modules function together as one primer $(2,3)$. This stacking effect was also described by O'Meara et al. (6) to enhance the capture of single-stranded DNA prehybridized with short oligodeoxynucleotides (ODNs) in positions next to the binding site of the capture probe. Unlabeled ODNs, designated "helpers," have also been used for in situ hybridization of rRNA, where a remarkable effect occurred when unlabeled ODNs hybridized adjacently to the labeled detection probe $(7,8)$. This was interpreted as an effect of the combination of base stacking and a reduction of the strong secondary structures of rRNA. These strong secondary structures are believed to be one of the major obstacles for the in situ hybridization of rRNA (9). Recently, the use of hairpin capture probes has shown that a probe containing a single-stranded region adjacently positioned to a duplex region hybridizes to targets with a thermodynamic advantage compared to regular linear probes $(10,11)$. This was believed to be attributed at least partly to stacking forces between the double-stranded region and the target sequence. Within the technology of ODN microarrays, the use of a "chaperone-detector probe strategy" has enhanced both hybridization specificity and signal intensity by hybridizing the detector probe adjacently to the capture probe, thereby facilitating the opening up of tertiary structures in the target (12).

In this study, the effects of unla- beled ODNs as helper probes have been investigated in a bead-based sandwich hybridization assay (13-16), using in vitro synthesized mRNA as a model target. The mRNA was simultaneously hybridized to capture probes immobilized to magnetic beads and detection probes labeled with a single digoxigenin (DIG) molecule. The two probes were complementary to two different positions on the RNA. Detection was achieved by binding of anti-DIGalkaline phosphatase, followed by enzymatic hydrolysis of the substrate p-aminophenol (pAP) phosphate, creating the electrode-reactive product pAP, which was redox-cycled on a chip with an interdigitated array electrode (16-18). The impact of the helpers on the hybridization efficiency was investigated with regard to the surface proximity of the beads, but the effects of base stacking and local secondary structures were considered. Finally, the method was used to detect mRNA species in total RNA extracts from Escherichia coli and Bacillus licheniformis.

\footnotetext{
${ }^{1}$ Novozymes A/S, Bagsvaerd, Denmark, ${ }^{2}$ Royal Institute of Technology KTH, Stockholm, Sweden, ${ }^{3}$ Polish Academy of Sciences,
} Gdansk, Poland, and ${ }^{4}$ The Technical University of Denmark, Lyngby, Denmark 


\section{MATERIALS AND METHODS}

\section{Oligonucleotides}

Purified ODNs were purchased from TAG Copenhagen (Copenhagen, Denmark). All ODN except those labeled with DIG were purified using a reversed phase column. Those labeled with DIG were purified by reverse phase high-performance liquid chromatography (HPLC) by the manufacturer, and all concentrations were as specified in the manufacturer's instructions. Table 1 lists all the probes and ODNs used in this study.

\section{Covalent Coupling of Amino-Linked Oligodeoxynucleotides to Magnetic Beads}

Carboxylated magnetic beads with a diameter of $2.8 \mu \mathrm{m}$ (Dynabeads ${ }^{\circledR}$ M-270 Carboxylic Acid; Dynal, Oslo, Norway) were bound to $5^{\prime}$-amino labeled ODN by incubation overnight at room temperature in the presence of the coupling reagents 1-ethyl-3-(3-dimethylaminopropyl) carbodiimide (EDCI) and 1-methylimidazole (both from Sigma-Aldrich Chemie GmbH, Steinheim, Germany) as previously described $(19,20)$. After the coupling reaction, the beads were washed three times with water to remove unbound probe and then three times with $0.1 \mathrm{M}$ sodium bicarbonate buffer, $\mathrm{pH} 8.0$, containing $0.1 \mathrm{M}$ ethanolamine (Sigma-Aldrich Chemie $\mathrm{GmbH})$ to block activated but unreacted carboxy groups. The beads were then washed three times with $1.0 \mathrm{M}$ sodium chloride (Sigma-Aldrich Chemie $\mathrm{GmbH}$ ) with $0.1 \%$ sodium dodecyl sulphate (SDS) (Sigma-Aldrich Chemie $\mathrm{GmbH}$ ) adjusted to $\mathrm{pH} 7.0$ to remove noncovalently bound ODN, followed by three washes with water. The beads were stored at $4^{\circ} \mathrm{C}$ in water treated with diethyl pyrocarbonate (DEPC) (SigmaAldrich Chemie $\mathrm{GmbH}$ ). All solutions were prepared in DEPC-treated water.
Immobilization efficiency was calculated from an absorbance at $260 \mathrm{~nm}$ of the reaction solution containing the ODN before and after the coupling reaction.

\section{Coupling of Biotin-Labeled Probes to Streptavidin Beads}

Streptavidin-coated magnetic beads with a diameter of $2.8 \mu \mathrm{m}$ (Dynabeads M-280 Streptavidin; Dynal) were used to immobilize biotin-labeled capture probes in experiments involving hybridizations to $16 \mathrm{~S}$ rRNA from $E$. coli. The probes were immobilized for $15 \mathrm{~min}$ at room temperature in Tris-buffered saline (TBS), using $0.5 \mu \mathrm{M}$ of probes in a bead suspension of $2.5 \mathrm{mg} / \mathrm{mL}$, and then washed in DEPC-treated water to remove unbound probes.

\section{RNA Purification}

RNA was extracted from bacterial cells growing in fed-batch fermentors 
Table 1. Sequences of All Oligonucleotide Probes Used in the Hybridizations

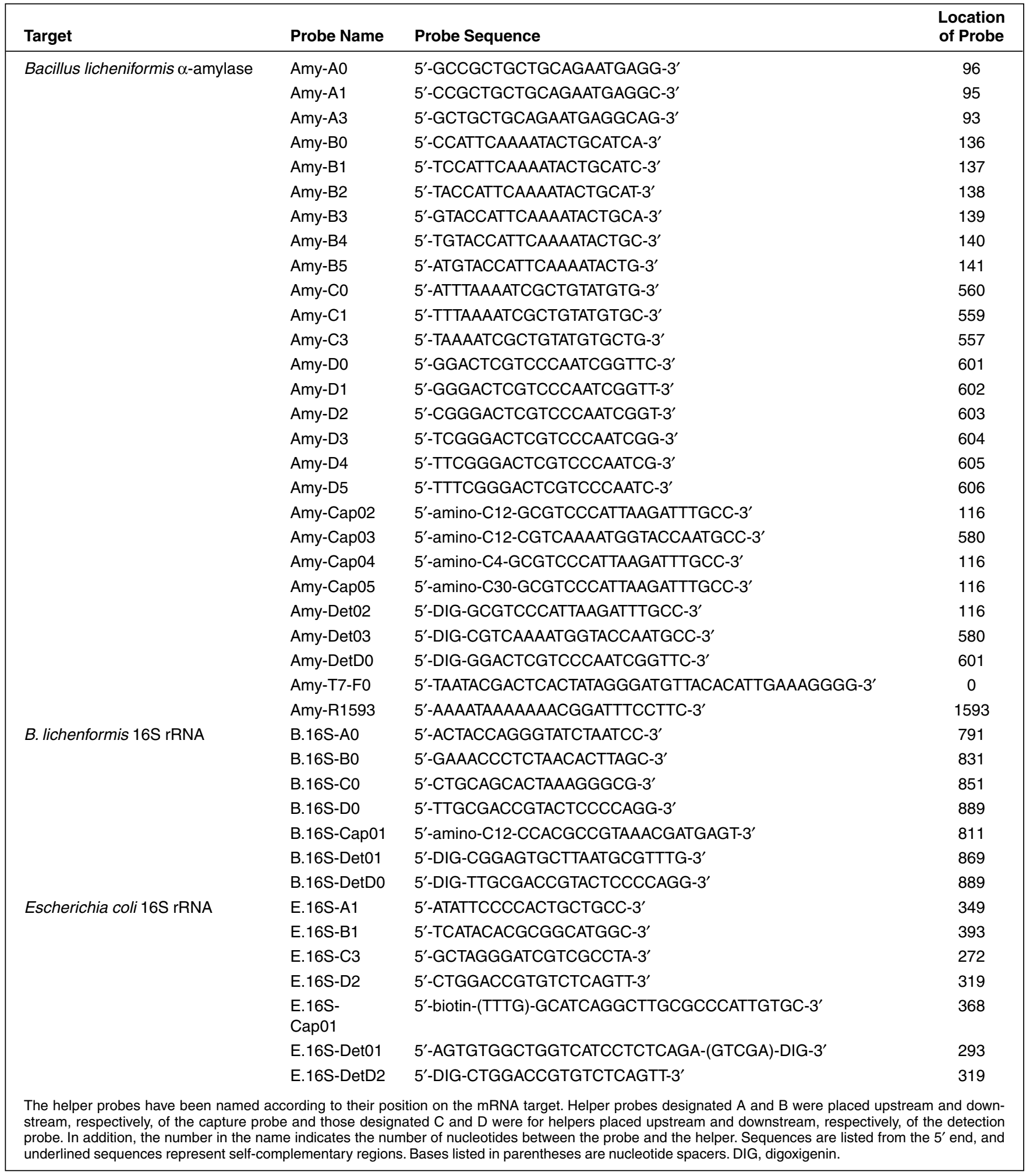

supplied with a complex-rich media. Cells were snap-frozen in Eppendorf ${ }^{\circledR}$ tubes (Eppendorf, Hamburg, Germany), held in a dry ice/ethanol bath and stored at $-80^{\circ} \mathrm{C}$. Cells were thawed in an ice water bath and 1 volume of TE buffer (10 mM Tris-Cl, $1 \mathrm{mM}$ EDTA, $\mathrm{pH} 8.0$ ) containing $5 \mathrm{mg} / \mathrm{mL}$ lysozyme and $4 \mathrm{mg} / \mathrm{mL}$ mutanolysin (Sigma-Aldrich Chemie $\mathrm{GmbH}$ ) and incubated for $1 \mathrm{~h}$ at $0^{\circ} \mathrm{C}$. RNA was then extracted using acid phenol as previously described 
(21). RNA was finally resuspended in DEPC-treated distilled water and quantified by UV absorbance.

\section{Sandwich Hybridization}

All sandwich hybridizations were performed in a $5 \times$ standard saline citrate (SSC) buffer containing $0.2 \%$ SDS and $2 \times$ Denhardt solutions (Sigma-Aldrich Chemie $\mathrm{GmbH}$ ). Beads (0.45 mg; $3 \times$ $10^{7}$ ) were prehybridized at $50^{\circ} \mathrm{C}$ in 100 $\mu \mathrm{L}$ of hybridization solution together with 5 pmol of DIG-labeled detection probe and 0-15 pmol of unlabeled helper ODN for $5 \mathrm{~min}$. The target RNA was then added, and hybridization continued for $1 \mathrm{~h}$ at $50^{\circ} \mathrm{C}$ in a Thermomixer (Eppendorf) at $1100 \mathrm{rpm}$. After the hybridization, the bead-bound sandwich complex was washed twice in $2 \times \mathrm{SSC}$ and $0.1 \% \mathrm{SDS}$ at $50^{\circ} \mathrm{C}$. The beads were washed in blocking buffer (DIG Wash and Block Buffer Set; Roche Diagnostics $\mathrm{GmbH}$, Mannheim, Germany) and then in blocking buffer containing 1:1000 anti-DIG-alkaline phosphatase FAB fragments (Roche Diagnostics $\mathrm{GmbH}$ ). After 30 min of enzyme complex binding, unbound enzyme was washed away in 4 washes with $1 \times \mathrm{SSC}$. All reactions were performed in Eppendorf tubes that were precoated for $15 \mathrm{~min}$ in a TBS buffer containing $3 \%$ bovine serum albumin (BSA) (Sigma-Aldrich Chemie $\mathrm{GmbH}$ ).

\section{Detection}

The bead-based sandwich complex labeled with alkaline phosphatase was incubated for $2 \mathrm{~h}$ at room temperature in TBS buffer, $\mathrm{pH}$ 8.0, containing 3 mM pAP phosphate (Universal Sensors, Kinsale-Sandycove, Ireland). The phosphate group was then hydrolyzed by alkaline phosphatase, and then the pAP could be measured. The supernatant was pumped over two interdigitated electrodes placed on a chip. A positive and a negative potential were applied to the electrodes, and the pAP was oxidized on the positive electrode to form quinoneimine, which could then be reduced at the negative electrode back to pAP. This redox-cycling produced an electrical current $(18,22)$ that was proportional to the pAP generated, and thus the amount of target RNA hybridized (16).
The chip is connected to a portable microprocessor-controlled potentiostat (ISIT, Itzehoe, Germany). Amperometric current in the range $2.5 \mathrm{pA}$ to 100 $\mathrm{nA}$ from both the positive and the negative electrode can be measured. An Ag/ $\mathrm{AgCl}$ reference electrode is positioned downstream in the flow from the chip, which is encapsulated in a small flow cell of $2.5 \mu \mathrm{L}$ volume. Data are transferred via a customized program to the analysis software Origin ${ }^{\circledR} 6.1$ (OriginLab, Northampton, MA, USA).

\section{In Vitro Transcription}

Full-length in vitro transcripts of $B$. licheniformis $\alpha$-amylase were prepared from PCR fragments containing the T7 promotor (GenBank ${ }^{\circledR}$ accession no. partial EMBL_A17930). The PCR fragment was then in vitro transcribed in $40 \mathrm{mM}$ Tris- $\mathrm{HCl}, \mathrm{pH} 8.0,15 \mathrm{mM}$ $\mathrm{MgCl}_{2}, 5 \mathrm{mM}$ dithiothreitol (DTT), and $50 \mu \mathrm{g} / \mathrm{mL}$ BSA, containing $1 \mathrm{mM}$ each of all four NTPs, $0.4 \mathrm{U} / \mu \mathrm{L}$ of T7 RNA polymerase (USB, Cleveland, $\mathrm{OH}$, USA), and $0.2 \mathrm{U} / \mu \mathrm{L}$ of SUPERase In $^{\circledR}$ RNase inhibitor (Ambion, Austin, TX, USA). The in vitro transcription was run for $2 \mathrm{~h}$ at $37^{\circ} \mathrm{C}$, and DNA was removed by an additional $15 \mathrm{~min}$ at $37^{\circ} \mathrm{C}$ with $0.9 \mathrm{U} / \mu \mathrm{L}$ of DNase (Roche Diagnostics $\mathrm{GmbH}$ ). Finally, the transcript was cleaned on RNeasy ${ }^{\circledR}$ columns (Qiagen, Hilden, Germany) according to the manufacturers' clean-up protocol and quantified by UV absorbance.

\section{RESULTS AND DISCUSSION}

In this study, a bead-based sandwich hybridization assay (15) with electrical detection on a chip (16) was used to investigate the effects of one or more unlabeled helper ODNs on detection sensitivity. Fuchs et al. (7) have applied it for in vitro hybridization, where a remarkable increase in hybridization efficiency was seen for RNA targets that previously showed low hybridization efficiency. These effects were believed to be a result of the reduced secondary structure in the area where the probe binds. This study supports this finding and further investigates helper probe design and its effects on hybridization efficiency. A schematic drawing of the sandwich hybridization 
principle using helpers is shown in Figure $1 \mathrm{~A}$ as well as the detection method (Figure 1B). The use of different heat denaturation procedures before hybridization (as opposed to adding helpers) did not show any significant effects on hybridization signal, nor did the addition of nonspecific ODN added to the hybridization solution, proving that the helper probes do not behave like nonspecific blockers.
We tested combinations of several different helpers, all designed to hybridize one nucleotide apart from the binding site of the probes. Significantly higher hybridization efficiencies were seen when the helpers hybridized next to the capture probe compared to helpers hybridized next to the detection probe (Figure 2). To investigate whether this was due to structural hindrance from

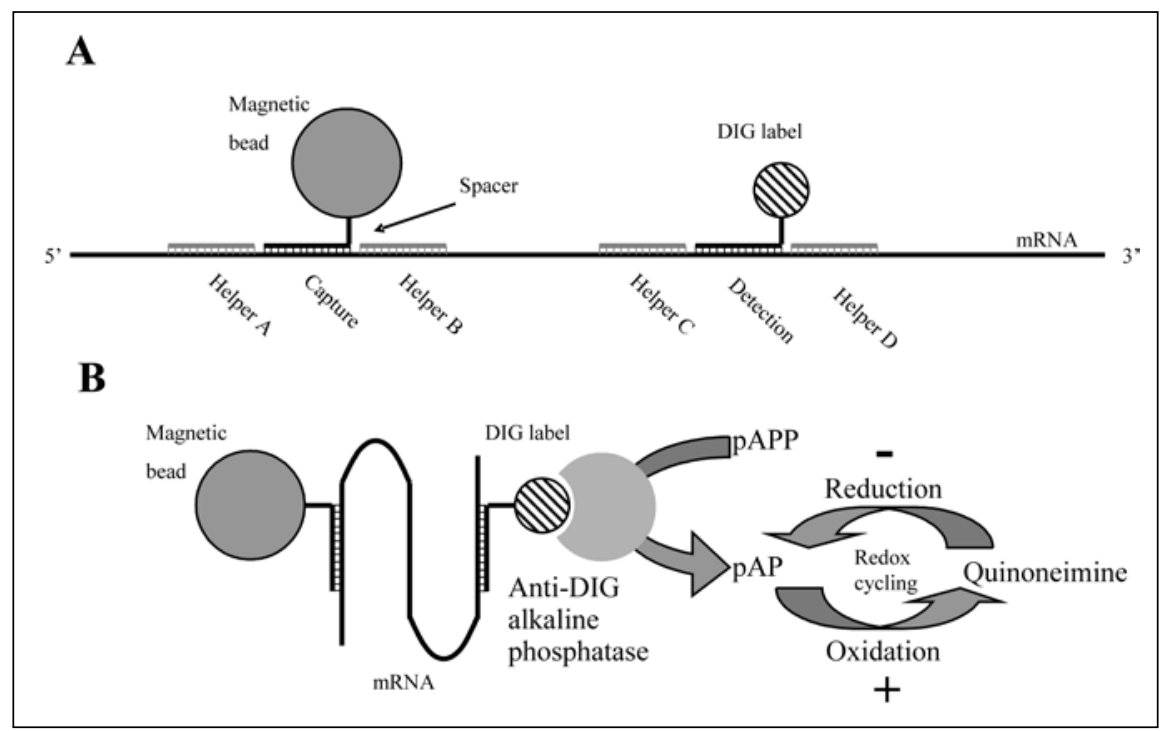

Figure 1. Illustration of sandwich hybridization and detection principle. (A) mRNA with capture, detection, and helper probe binding sites. The helper probes are named A, B, C, and D, respectively. (B) Illustration of detection principle on chip. Anti-digoxigenin (DIG) alkaline phosphatase binds DIG molecules, and then the enzyme converts p-aminophenol phosphate (pAPP) to the electrode-active compound p-aminophenol (pAP), which is redox-cycled at the electrodes, thereby creating a current.

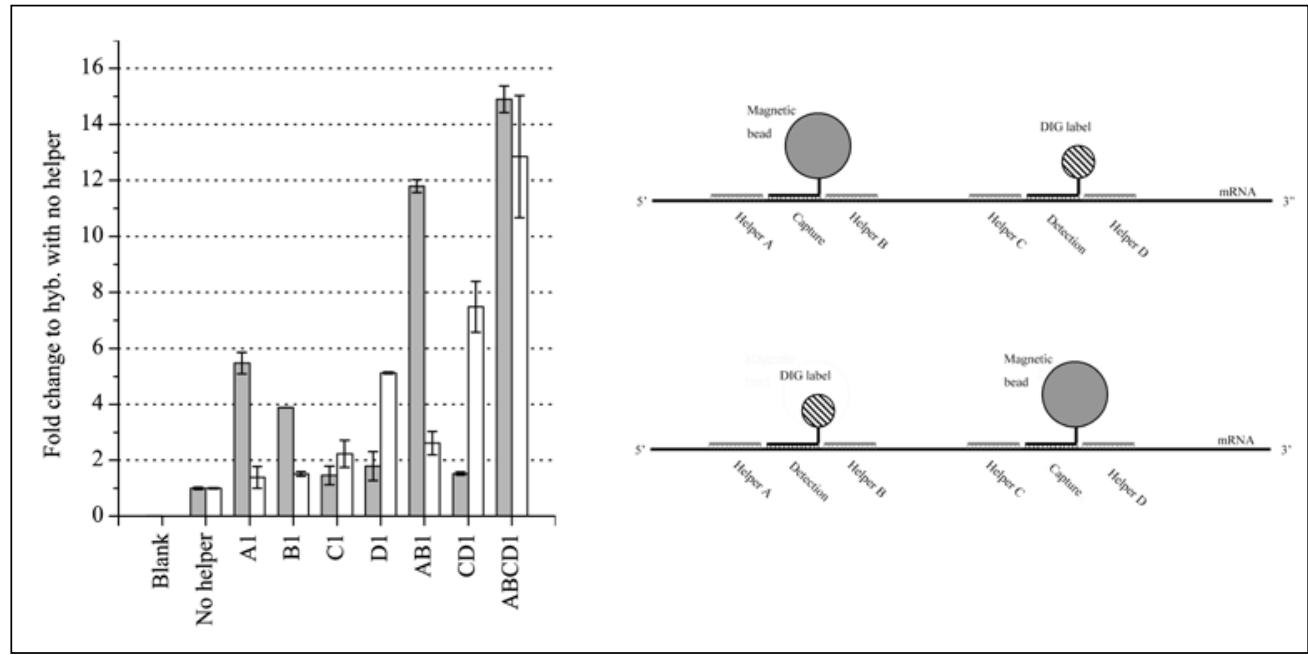

Figure 2. Effect of different helper probes on hybridization efficiency. Signals from hybridization with different helpers relative to the hybridization signal acquired from hybridization without helper probes, which is defined as 1 . The gray bars represent the hybridization of the capture and detection probes in their regular position (as shown in Figure 1). The white bars represent the results obtained from switching the positions of the capture and detection probes so that the detection probe binds adjacent to helpers $\mathrm{A}$ and $\mathrm{B}$ and the capture probe binds adjacent to $\mathrm{C}$ and $\mathrm{D}$. Helper concentration was $5 \times 10^{-8} \mathrm{M}$, and the target mRNA concentration was $2.5 \times 10^{-10} \mathrm{M}$ in all experiments. Data were normalized, and each bar is the mean of two independent experiments with the error bars representing the SD $(\overline{\times} \pm$ SD). the bead or reduced secondary structure around the target region of the detection probe in this particular mRNA molecule, we set up an experiment where the labels of the two probes were switched so that the bead-bound capture probe hybridized to the region where the detection probe hybridized before and vice versa. Figure 2 shows that when the capture and detection probes were switched, a significant drop in signal was seen when using the helper A alone, helper $\mathrm{B}$ alone, and the combination $\mathrm{AB}$. At the same time, an increase in signal was seen in switching experiments with helpers $\mathrm{C}, \mathrm{D}$, and $\mathrm{CD}$, indicating that the main effect of the helper ODN is to reduce structural and/or steric problems near the bead surface compared to a probe in solution. This is most likely a result of the reduced reaction range of the immobilized probe, which results in a smaller reaction volume that makes it difficult to reach the target sequence in the RNA. The increased effect of helpers when positioned adjacent to the probe immobilized to the bead could, aside from disrupting local secondary structures, also be a result of a disruption of tertiary structures. Mir et al. (23) have studied the tertiary structure effects in heteroduplex formation and found that in some cases tertiary structures in tRNA prevent the hybridization of ODN.

To examine this further, we prepared magnetic beads with different lengths of carbon spacers between the bead and the ODN. Spacers consisting of 4,12 , and 30 carbon atoms were tested. The measured immobilization efficiencies were $12.6,12.4$, and $10.7 \mu \mathrm{g}$ probe/mg beads, respectively. It was clear that the short four carbon spacer showed only very low hybridization efficiency compared to the two others without the use of helper probes. When adding the helper probes ABCD1, a significant increase in hybridization efficiency was seen for all three types of spacers, although the signal from the experiment with the four carbon spacer never reached the level seen with the two others. The effect of using helper probes de- 
creased with increasing spacer length (data not shown).

To investigate the effect of helper probe concentration, we performed hybridizations where all parameters were kept constant, except for the helper probe concentration. In these experiments, all four helpers complementary to the RNA strand and all with one nucleotide distance to the respective capture and detection probes were used. As expected, we observed increasing hybridization efficiency as a function of helper probe concentration until the reaction became saturated. Surprisingly, an increasing effect of the helpers was observed, even though the helpers were in more than 500-fold molar excess to the target in the hybridization reaction (data not shown).

The effect of helpers has been described as being dual $(24,25)$. They assist by opening up the secondary structure around the target where the probe is

to bind. Additionally, there is an effect of base stacking between two adjacent terminal nucleotides from two ODNs hybridizing next to each other. This effect appears when the planar faces of the bases interact and stabilize the connection between the two probes by weak van der Waals forces and dipole-dipole interactions formed between the bases of the adjacent nucleotides of the two probes (26). To study these effects, we tested the two helpers B and D with varying distances; similar experiments were also performed (Figure 3). For the helper close to the bead (helper B), a rather random effect from changing the distance was observed, suggesting that perturbations of the local secondary structures are important, whereas the helper next to the detection probe (helper D) did not show any significant effects. This apparent lack of stacking effects might be explained by the presence of the $5^{\prime}$ end DIG label next to helper $\mathrm{D}$, which reduces the stacking effect (27).

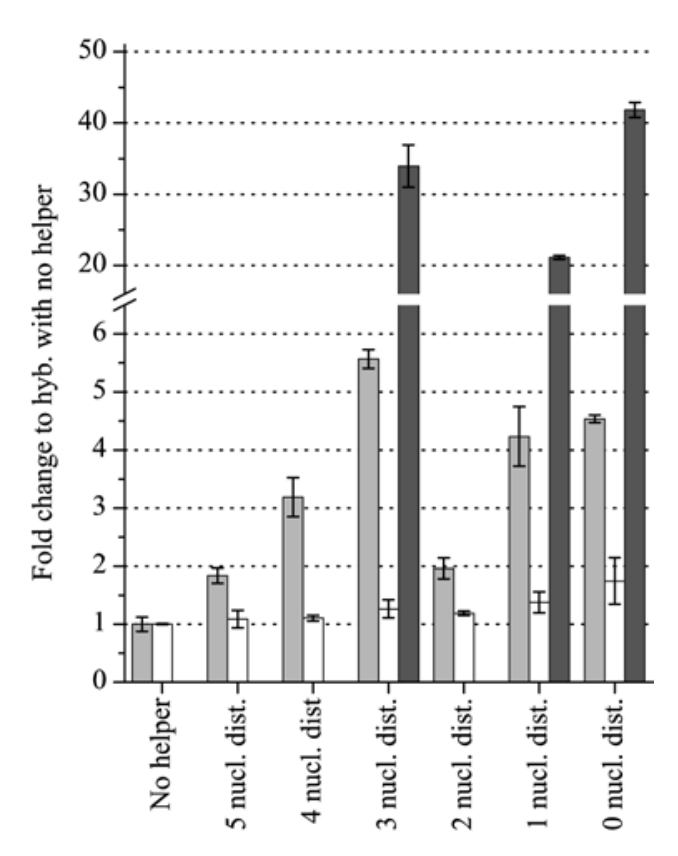

Figure 3. Comparison of hybridization signals from the hybridization of helpers with different distance to the capture and detection probes. Signals are relative to hybridization without helpers, which is defined as 1 . Light gray bars indicate results from experiments with helper B, white bars are from experiments with helper $\mathrm{D}$, and dark gray bars are from experiments when all helpers $\mathrm{ABCD}$ are used (tested only in three conditions but all with the same distance to the probe). The helper concentration was $10 \times 10^{-8} \mathrm{M}$, and the target mRNA concentration was $2.5 \times$ $10^{-10} \mathrm{M}$ in all experiments. Data were normalized, and each bar is the mean of two independent experiments with the error bars representing the SD $(\bar{x} \pm \mathrm{SD})$. Hyb, hybridization; nucl. dist., distance in nucleotides from the probe.
When using all four helpers, the highest signal was acquired when they were positioned at zero distance to the probes. However, when all four probes were positioned three nucleotides away, a greater effect was seen than when using probes positioned one nucleotide away from the probes. This indicates that local secondary structural effects play a major role in hybridization efficiency.

To show that the effects of helper probes were linear over the detection range of the method, we prepared two standard curves: one with and one without the addition of all four helper probes at zero distance from the capture and detection probes. To improve the detection limit, the hybridization time was extended to $2 \mathrm{~h}$. Initial experiments showed that this would improve hybridization efficiency (data not shown). As seen in Figure 4, the two curves are both linear and almost parallel. The detection limit of the method 


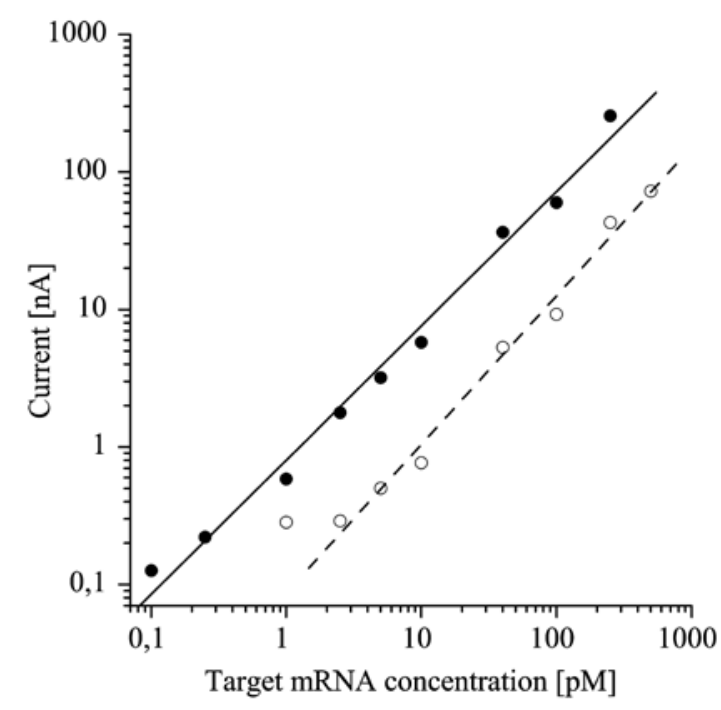

Figure 4. Amount of target mRNA molecules added to a reaction as a function of current measured on the chip in nanoampere (nA). Hybridization with (black circles) and without (open circles) $10 \times 10^{-8} \mathrm{M}$ of helper $\mathrm{ABCD} 0$ added per hybridization reaction. The hybridization time was extended to $2 \mathrm{~h}$. The lines are the calculated trend lines, under the assumption that the signal is linearly correlated to the amount of mRNA in the reaction.

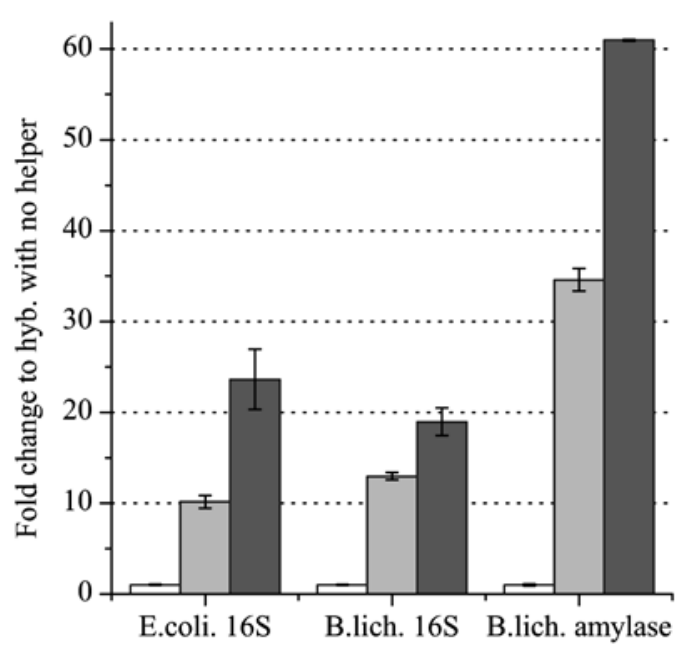

Figure 5. Hybridization to $16 \mathrm{~S}$ rRNA and $\alpha$-amylase mRNA from total RNA extracted from Escherichia coli (E.coli.) and Bacillus licheniformis (B.lich.) cells, respectively. Detection and helper probes $\left(10 \times 10^{-8} \mathrm{M}\right)$ used in the experiments. Comparison of hybridizations without helpers (white bars), with all four helpers (light gray bars), and with the addition of a digoxigenin (DIG)-labeled detection probe (dark gray bars), doubling the possible number of alkaline phosphatase enzymes per target molecule. The bars represent a fold increase compared to the experiments without helpers. Data were normalized, and each bar is the mean of three independent experiments, with the error bars representing the SD $(\overline{\times} \pm$ SD). was found to be less than $2 \times 10^{-13} \mathrm{M}$ mRNA molecules when all four helpers were added. The upper limit was not further examined because the instrument has a maximum detection limit of $100 \mathrm{nA}$, necessitating that samples with a higher amount of RNA target (more than approximately $1.5 \times 10^{-10}$ $M$ in the case of $\alpha$-amylase) be diluted before the measurement and was therefore more laborious. The extended hybridization time favored the hybridization without helper, so that the difference between hybridization with and without helpers was reduced to only 6- to 7-fold, but at the cost of time. The hybridization of the immobilized capture probe and RNA target is expected to be rate-limiting in the procedure, and by introducing unlabeled helper probes that are expected to have very fast hybridization kinetics (26), it would be possible to reduce the time required for the hybridization of the capture probe and RNA target because the helpers facilitate hybridization of the neighboring probe once bound. Increasing the hybridization time to $2 \mathrm{~h}$ will therefore reduce the difference in hybridization signal with and without helpers.

By performing rehybridization ex- periments of the same hybridization solution repeatedly to new beads, the hybridization efficiency was determined to be approximately $90 \%$ after $1 \mathrm{~h}$ in the presence of helper ABCD0 (data not shown).

To test the effect of helper probes with RNA targets extracted from bacterial cells, three RNA molecules were tested from two different strains: $16 \mathrm{~S}$ rRNA from E. coli and 16S rRNA and $\alpha$-amylase mRNA from $B$. licheniformis. We found that the helpers work with total RNA samples, and that the hybridization efficiency was increased more than 10-fold. By using an extra DIG-labeled detection probe complementary to the region where helper $\mathrm{D}$ is targeted, it was possible to increase the signal even further by allowing up to two alkaline phosphatases to bind to the same target. The huge effect seen with the helpers $\mathrm{ABCD} 0$ when hybridizing to the in vitro synthesized $\alpha$-amylase was repeated with RNA from a cellular source, where a more than 30-fold signal increase was observed (Figure $5)$. Using the system with two detection probes and helper probes, a 20- to 60fold signal increase was seen for rRNA and mRNA targets, compared to hy- bridizations with one detection probe and no helper probes. The experiments performed with $E$. coli RNA were done using streptavidin beads with biotinlabeled capture probes attached instead of the carboxylated surfaces that were used in all other experiments reported here. This demonstrates that the technique using helper probes works with capture probes attached to surfaces other than carboxylated ones.

The capture probe for the $\alpha$-amylase target was designed to be complementary to the loop region of a stem loop and thus $85 \%$ of the mRNA sequence was single-stranded under the hybridization conditions used, according to secondary structure prediction software at the Mfold Web server (28), and the adjacently positioned helper probes were targeting the double-stranded stem structure. It was therefore expected that the capture probe would bind with high efficiency without the use of helpers. The detection probe was complementary to a region in the RNA with only $50 \%$ single-stranded sequence. The pronounced effect of adding helper probes suggests that the opening of the stem loop structure alters the secondary and/or tertiary structure in a way that 
enhances hybridization. This effect is most prominent with the capture probe that is associated with a surface.

The greatest effect with bead-based sandwich hybridization was achieved by using all four helpers. However, using only two helpers adjacent to the capture probe showed almost the same effect. We therefore conclude that the phenomenon is primarily associated with hybridization to surface-attached ODN.

Huang et al. (29) recently showed that introducing unlabeled hairpin competitor probes complementary to the binding site of the detector probe increased the specificity of the hybridization. By combining these unlabeled hairpin competitor probes that target the binding site of the detection probe with helper probes complementary to sequences adjacent to the binding site of the capture probe, it would in theory be possible to obtain a method of high sensitivity and high specificity, without the use of nucleic acid amplification, which is difficult to perform unbiased.

\section{ACKNOWLEDGMENTS}

We thank Martin Persson (Novozymes $A / S$ ) for helpful discussions and critical reading of the manuscript. This work was supported by the European Research, Technological Development and Demonstration (RTD) project QLRT-1999-00533.

\section{REFERENCES}

1.Kieleczawa, J., J.J. Dunn, and F.W. Studier. 1992. DNA sequencing by primer walking with strings of contiguous hexamers. Science 258:1789-1791

2.Kotler, L.E., D. Zevin-Sonkin, I.A. Sobolev, A.D. Beskin, and L.E. Ulanovsky. 1993 DNA sequencing: modular primers assembled from a library of hexamers or pentamers. Proc. Natl. Acad. Sci. USA 90:4241-4245.

3.Kotler, L., I. Sobolev,and L. Ulanowsky. 1994. DNA sequencing: modular primers for automated walking. BioTechniques 17: 554-559.

4.Beskin, A.D., D. Zevin-Sonkin, I.A. Sobolev, and L.E. Ulanovsky. 1995. On the mechanism of the modular primer effect. Nucleic Acids Res. 23:2881-2885.

5.Nilsson, P., D. O'Meara, F. Edebratt, B. Persson, M. Uhlén, J. Lundeberg, and $P$. Nygren. 1999. Quantitative investigation of the modular primer effect for DNA and peptide nucleic acid hexamers. Anal. Biochem.
269:155-161.

6.O'Meara, D., P. Nilsson, P. Nygren, M. Uhlén, and J. Lundeberg. 1998. Capture of single-stranded DNA assisted by oligonucleotide modules. Anal. Biochem. 255:195-203.

7.Fuchs, B.M., F.O. Glöckner, J. Wulf, and R. Amann. 2000. Unlabeled helper oligonucleotides increase the in situ accessibility to $16 \mathrm{~S}$ rRNA of fluorescently labeled oligonucleotide probes, Appl. Environ. Microbiol. 66:3603-3607.

8.Dedysh, S.N., M. Derakshani, and W. Liesack. 2001. Detection and enumeration of methanotrophs in acidic Sphagnum peat by 16S rRNA fluorescence in situ hybridization, including the use of newly developed oligonucleotide probes for Methylocella palustris. Appl. Environ. Microbiol. 67:4850-4857.

9.Fuchs, B.M., K. Syutsubo, W. Ludwig, and R. Amann. 2001. In situ accessibility of Escherichia coli $23 \mathrm{~S}$ rRNA to fluorescently labeled oligonucleotide probes. Appl. Environ. Microbiol. 67:961-968.

10.Lane, M.J., T. Paner, I. Kashin, B.D. Faldasz, B. Li, F.J. Gallo, and A.S. Benight. 1997. The thermodynamic advantage of DNA oligoucleotide 'stacking hybridization' reactions: energetics of a DNA nick. Nucleic Acids Res. 25:611-616.

11.Riccelli, P.V., F. Merante, K.T. Leung, S. Bortolin, R.L. Zastawny, R. Janeczko, and A.S. Benight. 2001. Hybridization of single-stranded DNA targets to immobilized complementary DNA probes: comparison of hairpin versus linear capture probes. Nucleic Acids Res. 29:996-1004.

12.Small, J., D.R. Call, F.J. Brockman, T.M. Straub, and D.P. Chandler. 2001. Direct detection of 16S rRNA in soil extracts by using oligonucleotide microarrays. Appl. Environ. Microbiol. 67:4708-4716.

13.Ishii, J.K. and S.S. Ghosh. 1993. Bead-based sandwich hybridization characteristics of oligonucleotide-alkaline phosphatase conjugates and their potential for quantitating target RNA sequences. Bioconjug. Chem. 4:34-41.

14.Wicks, B., D.B. Cook, M.R. Barer, A.G. O'Donnell, and C.H. Self. 1998. A sandwich hybridization assay employing enzyme amplification for determination of specific ribosomal RNA from unpurified cell lysates. Anal. Biochem. 259:258-264.

15.Rautio, J., K.B. Barken, J. Lahdenperä, A. Breitenstein, S. Molin, and N. Neubauer. 2003. Sandwich hybridisation assay for quantitative detection of yeast RNAs in crude cell lysates, Microb. Cell Fact. 2:4.

16.Gabig-Ciminska, M., A. Holmgren, H. Andresen, K.B. Barken, M. Wümpelmann, J. Albers, R. Hintsche, A. Breitenstein, et al. Electric chips for rapid detection and quantification of nucleic acids. Biosens. Bioelectron. (In press.)

17.Hintche, R., M. Paeschke, U. Wollenberger, U. Schnakenberg, B. Wagner, and T. Lisec. 1994. Microelectrode arrays and aplication to biosensing devices. Biosens. Bioelectron. 9: 697-705.

18.Wollenberger, U., M. Paeschke, and R. Hintche. 1994. Interdigitated array microelectrodes for the determination of enzyme activities. Analyst 119:1245-1249.
19.Lund, V., R. Schmid, D. Rickwood, and E. Hornes. 1988. Assessment of methods for covalent binding of nucleic acids to magnetic beads, Dynabeads, and the characteristics of the bound nucleic acids in hybridization reactions. Nucleic Acids Res. 16:10861-10880.

20.Walsh, M.K., X. Wang, and B.C. Weimer. 2001. Optimizing the immobilization of single-stranded DNA onto glass beads. J. Biochem. Biophys. Methods 47:221-231.

21.Resnekov, O., L. Rutberg, and A. von Gabain. 1990. Changes in the stability of specific mRNA species in response to growth stage in Bacillus subtilis. Proc. Natl. Acad. Sci. USA 87:8355-8359.

22.Niwa, O., Y. Xu, H.B. Halsall, and W.R. Heineman. 1993. Small-volume voltametric detection of 4-aminophenol with interdigitated array electrodes and its application to electrochemical enzyme immunoassay. Anal. Chem. 65:1559-1563.

23.Mir, K.U. and E.M. Southern. 1999. Determining the influence of structure on hybridization using oligonucleotide arrays. Nat. Biotechnol. 17:788-792.

24.Maldonado-Rodriguez, R., M. EspinosaLara, A. Calixto-Suárez, W.G. Beattie, and K.L. Beattie. 1999. Hybridization of glass-tethered oligonucleotide probes to target strands preannealed with labeled auxiliary oligonucleotides. Mol. Biotechnol. 11:1-12.

25.Maldonado-Rodriguez, R., M. EspinosaLara, P. Loyola-Abita, W.G. Beattie, and K.L. Beattie. 1999. Mutation detection by stacking hybridization on genosensor arrays. Mol. Biotechnol. 11:12-25.

26.Anderson, M.L.M. 1999. Introduction to nucleic acid hybridization, p. 2-3; Hybridization strategy: oligonucleotide probes, $\mathrm{p}$ 77-87. In D. Rickwood (Ed.), Nucleic Acid Hybridization. Bios Scientific Publisher, Ltd., Oxford, UK.

27.Vasiliskov, V.A., D.V. Prokopenko, and A.D. Mirzabekov. 2001. Parallel multiplex thermodynamic analysis of coaxial base stacking in DNA duplexes by oligodeoxyribonucleotide microchips. Nucleic Acids Res. 29:2303-2313.

28.Zuker, M. 2003. Mfold web server for nucleic acid folding and hybridization prediction. Nucleic Acids Res. 31:3406-3415.

29.Huang, T.J., M. Liu, L.D. Knight, W.W. Grody, J.F. Miller, and C.-M. Ho. 2002. An Electrochemical detection scheme for identification of single nucleotide polymorphisms using hairpin-forming probes. Nucleic Acids Res. 30:e55.

Received 6 October 2003; accepted 28 October 2003.

\section{Address correspondence to}

Kim Bundvig Barken

Novozymes A/S

Smormosevej $251 J 14$

DK-2800 Bagsvaerd

Denmark

e-mail:sidsel_kim@email.dk 